\title{
Implicaciones sedimentológicas sobre el cambio en la cobertura del bosque de manglar en Boca Zacate, Humedal Nacional Térraba-Sierpe, Costa Rica
}

\author{
Ana Margarita Silva Benavides*1, 2 , Jorge Picado Barboza ${ }^{3}$, Fernando Mora Rodríguez ${ }^{4} \&$ \\ Carmen González Gairaud ${ }^{1}$ \\ 1. Escuela de Biología, Universidad de Costa Rica, San Pedro, San José 2060, Costa Rica; ana.silva@ucr.ac.cr, \\ carmenrgg@gmail.com \\ 2. Centro de Investigación en Ciencias del Mar y Limnología (CIMAR), Universidad de Costa Rica, San Pedro, San José \\ 2060, Costa Rica. \\ 3. Proyecto Hidroeléctrico El Diquís, Instituto Costarricense de Electricidad; JPicado@ice.go.cr \\ 4. Estudiante M.Sc. Gestión Integrada de Áreas Costeras Tropicales, Universidad de Costa Rica, 2009 - 2013; \\ fermorar@gmail.com \\ * Correspondencia
}

Recibido 30-IX-2014. Corregido 19-III-2015. Aceptado 20-IV-2015.

\begin{abstract}
Sedimentological implications of the change in the coverage of mangrove forest in Boca Zacate, Térraba-Sierpe National Wetlands, Costa Rica. In the last sixty years many geomorphological changes have occurred in Costa Rica's Térraba-Sierpe National Wetlands. Changes in coastal geomorphology are generally associated with erosion or accretion of sediment, which has led to the removal of sections of mangrove forests or sediment banks colonized by mangroves. The aim of this study was to analyze sedimentation as a leading process in the dynamics of coastal morphology and its implications for mangrove forest cover in the Boca Zacate area of Térraba-Sierpe wetlands. The study was conducted in the sectors of Bocón, Brujo and Coco Island in Boca Zacate, from 2008 to 2013. The research was based on a multi-temporal analysis of coastal morphology using aerial photographs from the years 1948, 1960, 1974, 1978, 1984, 1992 and 2011. The following measurements were also performed: monthly sedimentation rate $\left(\mathrm{g} / \mathrm{cm}^{2} /\right.$ day), and granulometric composition and content of chemical elements in the sediments of the study area. These last two measurements were performed once each in the dry and rainy seasons during the years of study. The results indicated that over the past 60 years, Boca Zacate has witnessed a process of sustained erosion; from 1948 through 2001, losing $10.6 \%$ of its land and approximately $8.9 \%$ of its forest cover. It has also experienced accretion in the area of Coco Island. The Brujo sector showed the highest sedimentation rate and the Camibar estuary, the lowest. The dominant type of sediment in all study sites was sand, followed by clay and silt. The most widespread chemical elements (mg/L) included magnesium, calcium and potassium; others, such as manganese, iron, aluminum, phosphorus, zinc and copper, were measured in smaller amounts. Transport, composition and quantity of sediment in Boca Zacate are crucial to the changes that have occurred on the coastal area of La Boca, where the presence of dead trees was evident. This geomorphological analysis holds great importance for future guidelines and actions for the conservation and integrated management of the mangrove in Terraba- Sierpe National Wetlands. Rev. Biol. Trop. 63 (3): 591-601. Epub 2015 September 01.
\end{abstract}

Key words: litoral morphodinamic, sediments, mangrove forest, Térraba Sierpe National Wetland, Costa Rica.

Los ecosistemas costeros se caracterizan por constituirse en espacios dinámicos que poseen una estrecha relación con el tipo, cantidad de sedimentos y su depósito por medio de la acción de agentes fluviales y marinos; ocasionando la colonización de nuevos bancos de sedimentos durante procesos de acreción o progradación litoral (Woodroffe, 1992).
A nivel mundial, estos acontecimientos son más visibles en sitios como los deltas y estuarios, donde la dinámica litoral está influenciada por el oleaje, las mareas y la descarga fluvial, esta última, con el aporte de sedimentos provenientes del continente (Arche, 1989 \& Elliot, 1991). Otros factores que dinamizan eventualmente la morfodinámica litoral 
están asociados con la sismicidad y la tectónica, donde el fallamiento local y la subducción ocasionan el basculamiento y la migración de cuerpos hídricos (Gutiérrez, 2008).

Una de las funciones más importantes de los ambientes de manglar presentes en sistemas deltaicos es el proveer un mecanismo de captación de sedimentos en las áreas costeras (Woodroffe, 1992 \& Kathiresan, 2003). Especies de mangle como Rhizophora mangle y Rhizophora racemosa capturan sedimentos por medio de su compleja estructura de raíces aéreas (Bird \& Barson, 1977), permitiendo de esta manera la progradación del delta. Sin embargo, un exceso de material sedimentológico en áreas de manglar puede causar la muerte de los árboles debido al sofocamiento de las raíces (Jiménez, Lugo, \& Cintrón, 1985, Ellison, 1998). Existen evidencias de que los procesos erosivos y la alta sedimentación relacionada con la acción de las olas y las mareas, han contribuido con cambios en el litoral y en los bosques de mangle en sitios como Australia, San Andrés, Filipinas y Benín (Blasco, Saenger, \& Janodet, 1996; González, Urrego, Martínez, Polanía, \& Yokoyama, 2010).

El delta Térraba-Sierpe se caracteriza por mantener una intensa dinámica reflejada en procesos de erosión y progradación de sus frentes de playa, donde la forma y tamaño de algunos sectores del bosque de mangle han variado, conllevando en algunos casos a la desaparición total de la vegetación. Basados en la premisa anterior, en el presente artículo se analiza la sedimentación como proceso importante en la dinámica de la cobertura del bosque de mangle en Boca Zacate, Humedal Nacional Térraba-Sierpe (HNTS).

\section{MATERIALES Y MÉTODOS}

Área de estudio: La cuenca del río Grande de Térraba constituye la cuenca más grande de Costa Rica, fluye hacia la costa Pacífica drenando un área de $5085 \mathrm{~km}^{2}$ (Cedeño, López, Villalobos, \& Hernández, 2012, Rojas, 2011). El sitio de estudio comprende el sector de Boca Zacate en el sistema deltaico Térraba-Sierpe y se localiza en el Humedal Nacional TérrabaSierpe en el Pacífico Sur, Costa Rica (Fig. 1 A y Fig. 1 B). El sector de Boca Zacate presenta en sus frentes de playa zonas de manglar muerto, y en la parte posterior prevalece acumulación de arena que provoca el deterioro de los árboles de mangle. Detrás de estas zonas se encuentra el bosque de mangle sano cuyas especies dominantes son Rhizophora racemosa y Pelliciera rhizophorae, con árboles que poseen alturas entre 20 y $40 \mathrm{~m}$.

Análisis de fotografías aéreas e imágenes satelitales: Con el propósito de sistematizar los cambios espacio-temporales en la morfología litoral y la cobertura del bosque, se digitalizó la línea de costa, la cobertura del bosque de mangle y los bancos de sedimento en cada una de las fotografías aéreas e imágenes satelitales de 1948, 1960, 1974, 1978, 1984, 1992, 1997 , 2003 y 2011 en los siguientes sectores de Boca Zacate: los esteros Bocón y Brujo, isla Mero, isla Coco (conocido como El Turco), islas Las Loras e isla Zacate. Para cada una de las fotografías digitalizadas, se construyó una matriz de análisis que consistió en obtener el área en $\mathrm{km}^{2}$, el porcentaje de la cobertura del bosque de mangle y de los bancos de sedimentos presentes durante el periodo estudiado. Este procedimiento se apoyó en la plataforma tecnológica para SIG, con el empleo del software Arc Gis 10 de Esri.

Tasa de sedimentación: Para cuantificar la tasa de sedimentación se tomaron muestras mensuales de sedimentos durante 2008-2013 en el canal principal de los esteros Bocón

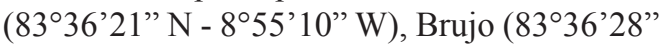
$\left.\left.\mathrm{N}-8^{\circ} 55>14^{\prime \prime} \mathrm{W}\right)\right)$, Estirahuevos (833' $53^{\prime \prime}$ N - 8०57>24” W) ) y Camíbar $\left(83^{\circ} 35^{\prime} 53^{\prime \prime} \mathrm{N}\right.$ $8^{\circ} 57>24$ ” W) ) (Fig. 1A). Se colocaron réplicas de tres trampas construidas de pvc de $50 \mathrm{~cm}$ de largo y $5 \mathrm{~cm}$ de diámetro en tres sitios a lo largo de cada estero: posterior (hacia el extremo continental), medio y anterior (hacia el extremo marino), sujetándolas a las raíces de los árboles de mangle para asegurarse que las trampas se cubrieran de agua durante la marea alta; 

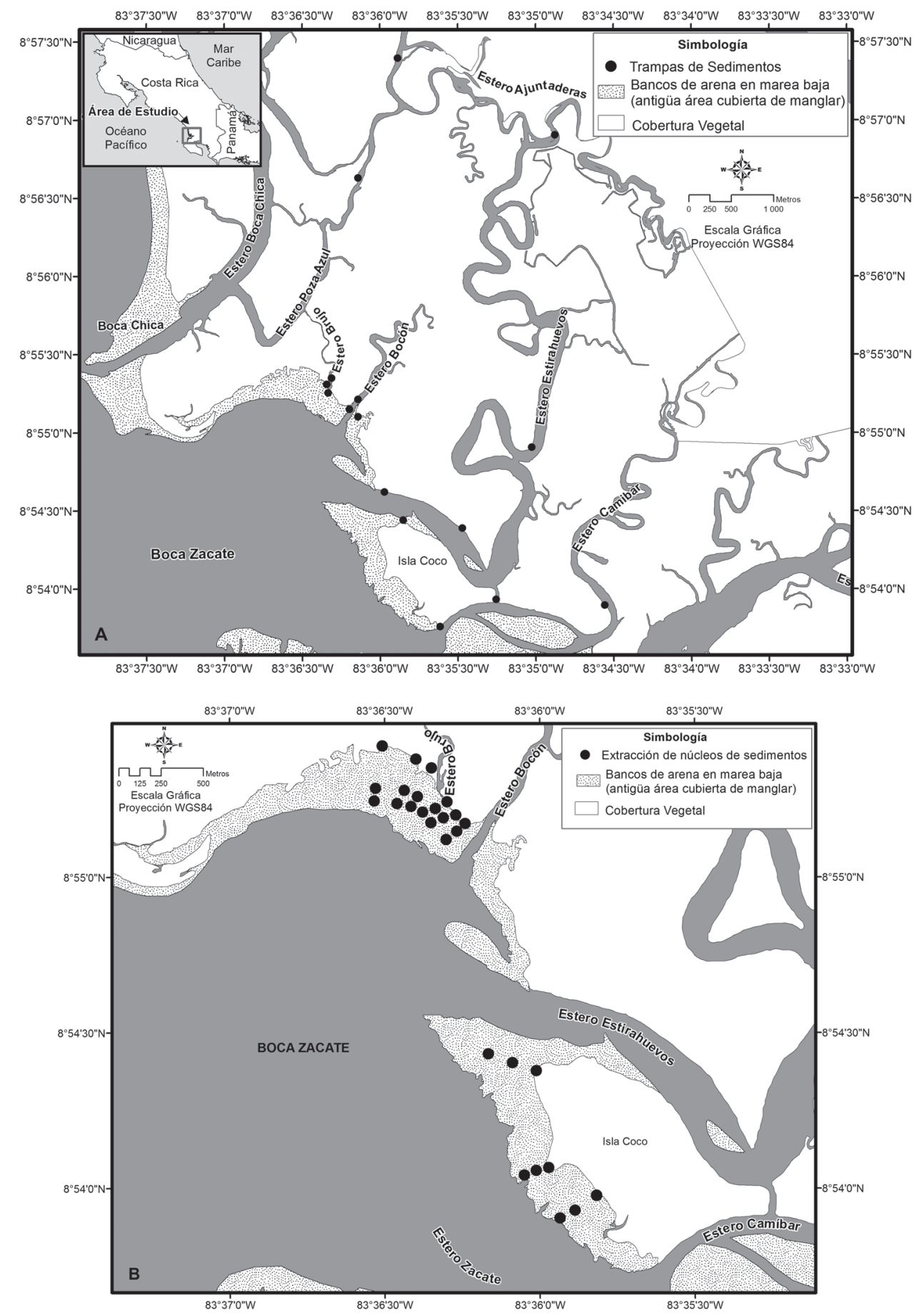

Fig. 1 A-B. Sitios de muestreo: (1A) trampas de sedimentos en los canales principales de los esteros y (1B) extracción de núcleos de sedimentos. Boca Zacate, Humedal Nacional Térraba Sierpe, Costa Rica. 2008-2013.

Fig. 1 A-B. Sampling sites: (1A) sediment traps in the main channels of estuaries and (1B) extraction of sediment cores. Boca Zacate, Térraba-Sierpe National Wetlands of Costa Rica. 2008-2013. 
además se colocó una trampa en el centro del canal principal la cual permaneció sumergida la mayor parte del tiempo entre los cambios de las mareas. Adicionalmente, se ubicaron un total de 4 trampas control fuera del área de estudio de los esteros evaluados. El contenido de las trampas de sedimentos fue recolectado cada 30 días. La tasa de sedimentación se expresó como $\mathrm{g} / \mathrm{cm}^{2} /$ día y corresponde a los gramos de sedimento capturado por el área de la apertura de la trampa $\left(20.26 \mathrm{~cm}^{2}\right)$, durante el número de días que permaneció la trampa ubicada en cada sitio de estudio.

\section{Composición granulométrica del sedi-} mento: Los análisis granulométricos se realizaron en los sedimentos recolectados en los canales de los esteros descritos anteriormente; además en los siguientes sectores de playa: Bocón (8336'9" N - 8 85 $^{\circ} 13^{\prime \prime}$ W), Brujo (8336'19" N - 8 $\left.8^{\circ} 55^{\prime} 21^{\prime \prime} \mathrm{W}\right)$ e isla Coco (8336'19" N - 8 $55^{\prime} 21^{\prime \prime}$ W) y sitios de control (C. Bocón: 855'22.69” N - 83³6’0.91” W; C. Brujo: $8^{\circ} 55^{\prime} 29.92 \mathrm{~N}-83^{\circ} 36^{\prime} 22.03^{\prime \prime} \mathrm{W}$ y C. Coco: $\left.8^{\circ} 54^{\prime} 27.97^{\prime \prime} \mathrm{N}-83^{\circ} 35^{\prime} 10.14^{\prime \prime} \mathrm{W}\right)$ en época seca y lluviosa durante 2008-2013 (Fig. 1A y Fig. 1B). En cada sitio, se realizaron tres perfiles horizontales perpendiculares a la línea de costa separados por una distancia entre 200 y $400 \mathrm{~m}$, marcados desde el punto más bajo descubierto en marea baja $\left(\mathrm{H}_{0}\right)$, un punto intermedio $\left(\mathrm{H}_{1}\right)$, hasta el punto más alto $\left(\mathrm{H}_{2}\right)$; correspondiente a la berma, separados por una distancia entre 150 y 200 m cada uno. En cada punto se tomaron muestras de sedimentos a las siguientes profundidades: $0-40,40-80$ y $80-120$ $\mathrm{cm}$ con un nucleador de PVC de $1.20 \mathrm{~m}$ de largo y $5.00 \mathrm{~cm}$ de diámetro.

Las muestras de sedimentos se secaron al horno a una temperatura de $100{ }^{\circ} \mathrm{C}$ durante 12 horas y se tamizaron con mallas de diferentes tamaños. Las partículas mayores a 0.0625 $\mathrm{mm}$ se clasificaron en el ámbito de las arenas y menor a ese tamaño como arcillas (Adams, MacKenzie, \& Guilford, 1984). Para el cálculo del porcentaje de cada fracción se analizó una muestra total de $100 \mathrm{~g}$, se separó cada una de las fracciones y se reportó la cantidad (\%) con respecto al peso total de la muestra según los procedimientos de Ellison (1998).

\section{Composición química del sedimento:} De los sedimentos recolectados para el análisis granulométrico se cuantificaron las concentraciones de los siguientes elementos químicos: hierro, calcio, potasio, cobre, magnesio, manganeso, fósforo, zinc, según la metodología propuesta por Olsen, Cole, Watanbe \& Dean (1954) modificada con KCL $1 \mathrm{~N}$.

Se realizaron análisis de varianza para evaluar el efecto de la estacionalidad sobre la tasa promedio de sedimentación, la composición granulométrica y elementos químicos de los sedimentos. La comparación de la composición granulométrica y química de los núcleos extraídos en los esteros Bocón, isla Coco y Brujo con sus respectivos núcleos control, fue realizada mediante la prueba no paramétrica Kruskal-Wallis. Todas las rutinas de análisis se realizaron utilizando el programa estadístico Statistica 6.

\section{RESULTADOS}

Análisis de fotografías aéreas e imágenes satelitales: En las formas de relieve litoral de Boca Zacate se observa una disminución de la superficie de $44.98 \mathrm{~km}^{2}$ en 1948 a $40.18 \mathrm{~km}^{2}$ en 2011 , lo que significa una pérdida de $4.79 \mathrm{~km}^{2}$ (11\%) durante 60 años. El área de cobertura vegetal ha disminuido de $41.98 \mathrm{~km}^{2}$ en 1948 a $38.25 \mathrm{~km}^{2}$ en el 2011, para un total de $3.73 \mathrm{~km}^{2}$ $(9.83 \%)$ durante el período de análisis.

En 1948, Boca Zacate mostró una boca reducida y con la zona de descarga hacia el norte. Para el 2011, se caracterizó por presentar una morfología abierta, con un mayor aporte de energía del oleaje y con la exposición de sectores de playa y un espacio de acreción dominado por arenas y vegetación de playa al norte de la Boca, que corresponde al sector estero Brujo.

El sector estero Bocón sufrió una erosión del $31 \%$ de la cobertura de mangle. Por su parte, el sector isla Mero, presentó una acreción intensa en el sector este, que se ha unido al resto del continente adyacente a la isla Coco, 
favoreciendo la colonización del manglar, principalmente por Rhizophora racemosa.

El sector isla Coco ha sufrido una pérdida de $59 \%$ de cobertura de bosque de mangle entre 1948 y 2011. En 1948, se observó una cobertura vegetal de $2.12 \mathrm{~km}^{2}$ y para 2011 se estimó en $867 \mathrm{~km}^{2}$. El cambio con mayor intensidad se presentó entre 1974 y 1984, con una erosión de $34 \%$. El sector isla Zacate mostró una disminución de la cobertura del bosque desde $4.70 \mathrm{~km}^{2}$ en 1948 a $2.50 \mathrm{~km}^{2}$ para el 2011, que correspondió a un $46 \%$ de pérdida del bosque. Estas variaciones se observan en la figura $2 \mathrm{~A}$ y figura $2 \mathrm{~B}$.

Tasa de sedimentación en el canal principal de los esteros: Se presentaron diferencias significativas en la tasa de sedimentación entre las épocas del año $\left(\mathrm{F}_{1 / 438}=8.5, \mathrm{p}<0.05\right)$, con el mayor aporte de sedimentos en la época lluviosa (promedio $0.8 \pm 0.6 \mathrm{~g} / \mathrm{cm}^{2} /$ día). Se cuantificaron diferencias significativas entre los esteros de estudio $\left(\mathrm{F}_{3 / 438}=39.2, \mathrm{p}<0.001\right)$, donde la mayor tasa de sedimentación se cuantificó en el canal principal del estero Brujo con un promedio de $(1.2 \pm 0.7) \mathrm{g} / \mathrm{cm}^{2} /$ día. Se observó un aumento de un $40 \%$ de sedimentos en las trampas ubicadas en el centro del canal principal con relación a las trampas ubicadas entre las raíces de los mangles, que se encuentran en las márgenes del canal $\left(\mathrm{F}_{1 / 118}=10.1, \mathrm{p}=0.001\right)$.

Composición granulométrica de los sedimentos en el canal principal de los esteros: La arena fue el tipo de sedimento que se cuantificó en mayor cantidad en los canales principales de los esteros, luego la arcilla y en menor proporción el limo, presentándose variaciones entre las épocas seca y lluviosa. La arena evidenció una mayor presencia durante la época lluviosa $\left(\mathrm{F}_{1 / 229}=8.03, \mathrm{p}<0.05\right)$; mientras que los limos y arcillas predominaron durante la época seca, sin embargo, en cantidades menores que la arena.

Composición granulométrica de los sedimentos en los sectores de playa Bocón, Brujo, isla Coco y sitios control: La arena fue el sustrato predominante en los sitios de estudio, especialmente en el perfil superior en la berma $\left(\mathrm{H}_{2}\right)\left(\mathrm{F}_{12 / 566,48}=3.19, \mathrm{p}<0.05\right)$; sin embargo, se observaron diferencias significativas en la composición granulométrica entre los sectores de muestreo $\left(\mathrm{F}_{15 / 1585}=28.28, \mathrm{p}<0.005\right)$ : la arena predominó en los sectores evaluados (Brujo, Bocón e isla Coco), mientras que en los sitios de control, los limos y arcillas constituyeron el sustrato dominante (Fig. 3).

Con relación a la época del año, se presentaron diferencias en la composición granulométrica de los sedimentos $\left(\mathrm{F}_{3 / 214}=12.61, \mathrm{p}<\right.$ $0.05)$. Prevaleció una leve tendencia a un incremento de las arenas y los limos en la época seca. Las arcillas aumentaron ligeramente durante la temporada lluviosa; sin embargo, en ambas épocas se mantuvo la predominancia de arenas en los sedimentos (Fig. 4A y Fig. 4B).

Con respecto a la composición granulométrica del sedimento en los perfiles de profundidad (40-80-120 cm), no se encontraron diferencias significativas $\left(\mathrm{F}_{30 / 1685,5}=0.88, \mathrm{p}\right.$ $>0.05)$, donde la arena fue el sustrato predominante en todas las profundidades evaluadas.

Composición química de los sedimentos: El magnesio $(\mathrm{Mg})$, calcio $(\mathrm{Ca})$, potasio $(\mathrm{K})$ son los elementos químicos dominantes en el sedimento de los canales y en los sectores Bocón, Brujo, isla Coco y sitios de control, mientras que el manganeso $(\mathrm{Mn})$, hierro $(\mathrm{Fe})$ y aluminio ( $\mathrm{Al})$, fósforo $(\mathrm{P})$, zinc $(\mathrm{Zn})$ y cobre $(\mathrm{Cu})$ se cuantificaron en cantidades menores. Los resultados muestran una alta variabilidad dentro y entre los sitios evaluados, todos los elementos químicos presentaron una mayor concentración promedio en el estero Camíbar, con la excepción del aluminio y el hierro que fueron más abundantes en el estero Bocón $(\mathrm{p}<0.05)$.

Si se comparan los sitios de la playa, se observa que la mayor concentración de calcio, magnesio y potasio $(\mathrm{mg} / \mathrm{L})$ se observó en el sector el Brujo, mientras que el fósforo, el zinc, el hierro y el aluminio fueron más abundantes en el sector de isla Coco $(\mathrm{p}<0.05)$. Todos los elementos químicos presentaron valores 

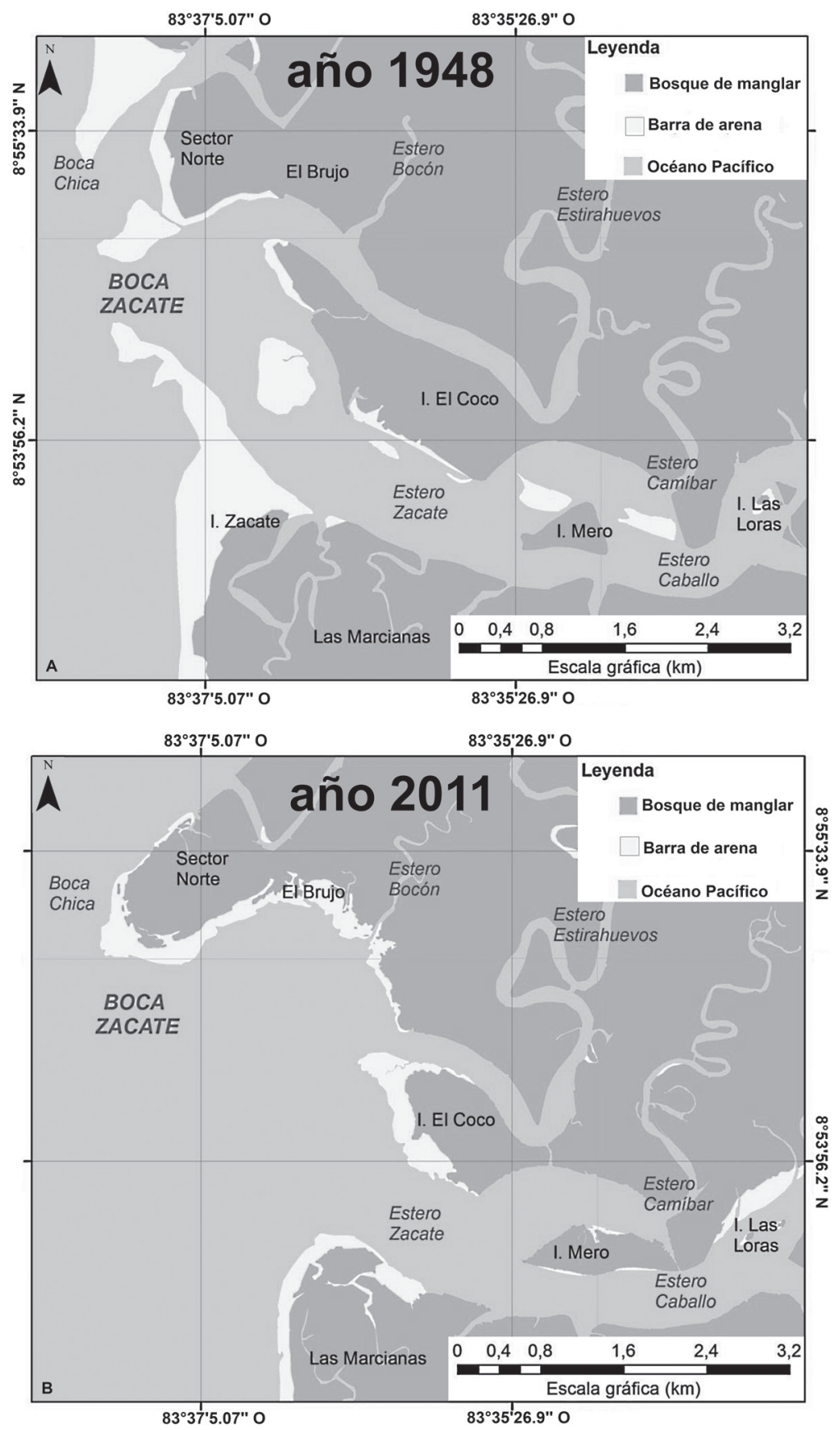

Fig. 2 A-B. Variaciones en la morfología litoral en Boca Zacate en 1948 (1A) y 2011 (1B). Sector estuarino del Humedal Nacional Térraba Sierpe, Costa Rica.

Fig. 2 A-B. Variations on the coastal morphology in Boca Zacate 1948 (1A) and 2011 (1B). National Estuarine Sector Térraba Sierpe, Costa Rica. 


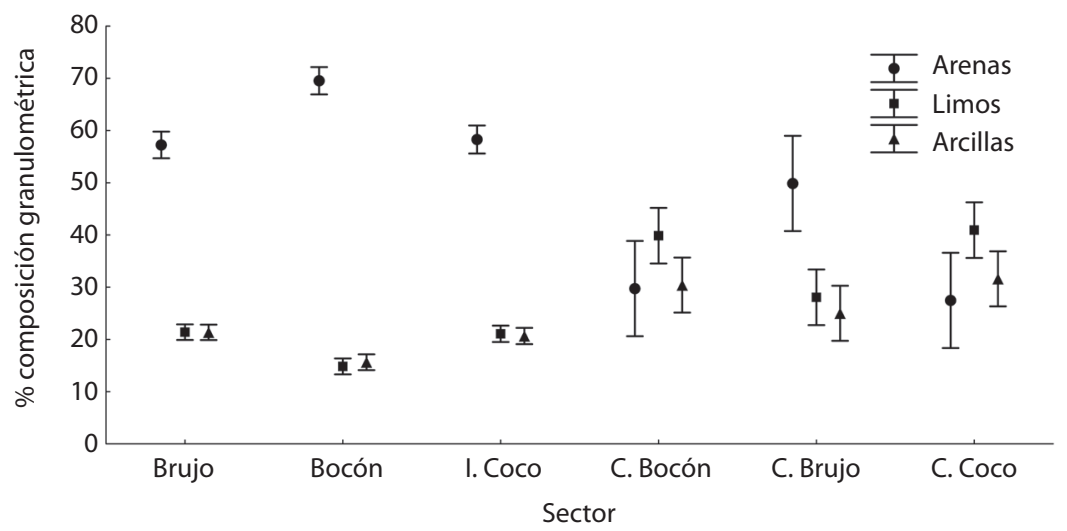

Fig. 3. Porcentaje de la composición granulométrica en cada sector de estudio. I. = isla, C. = control. Boca Zacate, Humedal Nacional Térraba Sierpe, Costa Rica. 2008-2013.

Fig. 3. Percentage of grain size in each sector of study. I. = isla, C. = control. Boca Zacate, Térraba Sierpe National Wetland, Costa Rica. 2008-2013.

bajos en los sitios donde predominaron las arenas $(\mathrm{p}<0.05)$.

\section{DISCUSIÓN}

La estructura y composición de los ecosistemas de manglar son influenciadas por gradientes ambientales, procesos físicos fluviales y litorales, mostrando una importante relación con la geomorfología de las costas (Woodroffe, 1992). Bajo esta óptica, la vegetación del manglar puede cambiar si la zona costera donde se encuentra sufre erosión o progradación. Estos procesos han sido reportados en varios bosques de manglares en Trinidad y Tobago, Singapur, Micronesia (Ellison, 1998), Colombia (Blanco, Estrada, \& Urrego, 2012) y Costa Rica (Lizano \& Salas, 2001; Cárdenes, 2003; Denyer, Cárdenes, \& Kruse, 2004; Lizano \& Lizano (2010) y Lizano (2013).

La distribución, composición y cantidad de sedimentos es determinante en un bosque de mangle, donde las raíces de los árboles y pneumatóforos constituyen eficientes trampas de sedimentos (Alongi, 2002), conformándose de esta manera áreas de rápida progradación (Woodrofe, 1992). En Boca Zacate, intervienen dos procesos estrechamente vinculados al aporte de sedimentos, uno de origen fluvial dominado por el río Grande de Térraba y otro litoral donde intervienen las corrientes, las mareas y el oleaje. Lizano (2012), menciona que las corrientes marinas en la zona costera del HNTS se dirigen hacia el norte durante la marea baja y hacia el sur en marea alta.

En nuestro contexto de estudio durante la marea alta, las corrientes se desplazan paralelas a los sectores Brujo, Bocón e isla Coco que se encuentran hacia el sur de la desembocadura del río Grande de Térraba, el cual durante períodos de aguas altas, contribuye con un aporte significativo de sedimentos hacia el sector litoral. Estos sedimentos son redistribuidos por las corrientes y el oleaje a lo largo de la línea de costa, dinámica que contribuye a modificar la morfología litoral y sistemas como el bosque de mangle que se encuentra en el sector.

Los mecanismos de transporte de sedimentos por parte de los esteros en un sistema deltaico, facilitan una entrada neta de sedimentos finos al bosque de mangle (Furukawa \& Wolanski, 1996). En el presente estudio, los esteros estudiados tienen una influencia directa del río Grande de Térraba; donde el estero Estirahuevos constituye la conexión principal. Este estero es meándrico y da origen a otros esteros como Bocón y Azul, este último a su vez origina el estero Brujo. Estos esteros transportan 


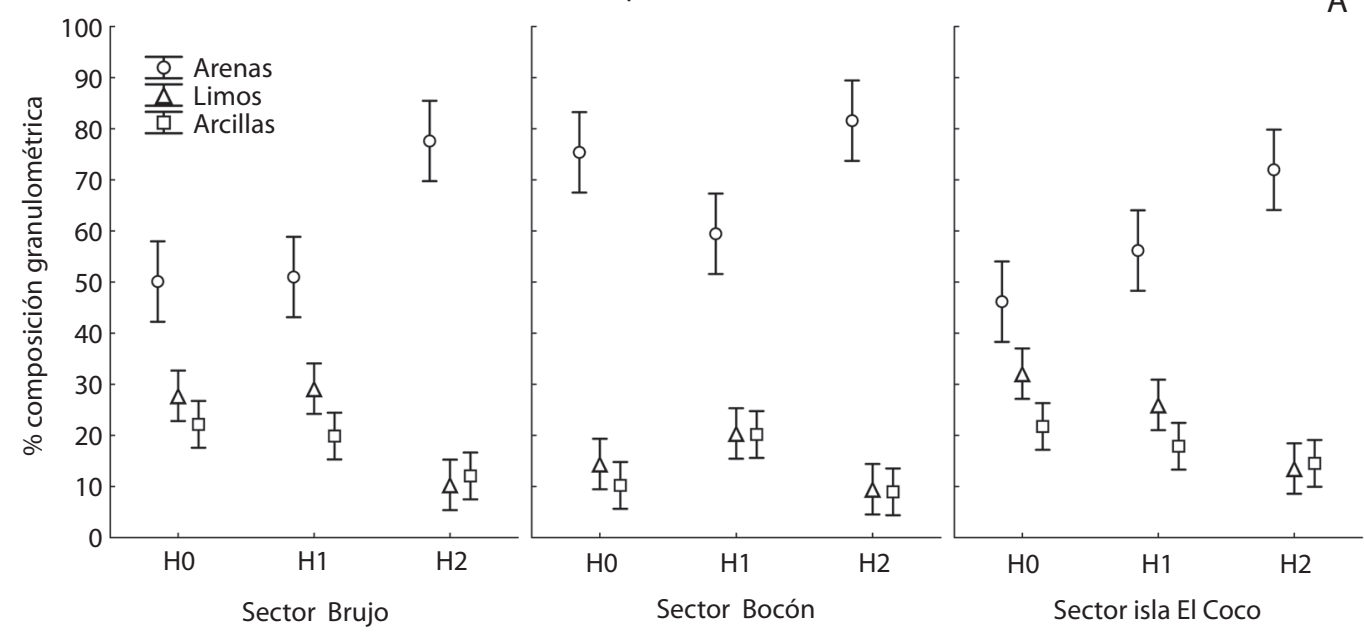

Época lluviosa
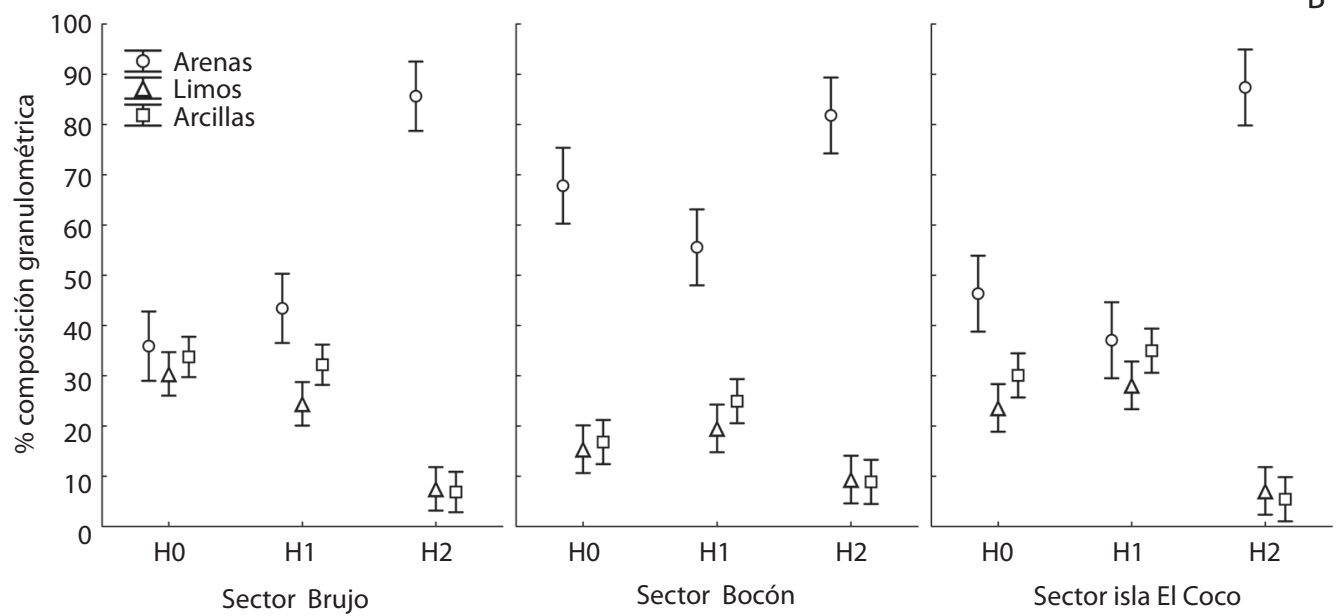

Fig. 4 A-B. Distribución de las arenas, limos y arcillas en la época seca y lluviosa en los sitios Bocón, Brujo e isla Coco y en los correspondientes perfiles (línea de costa H0, parte media H1 y parte posterior H2). Boca Zacate, Humedal Nacional Térraba Sierpe, Costa Rica. 2008-2013.

Fig. 4 A-B. Distribution of sands, silts and clays in the dry and rainy seasons in Bocón, Warlock and island Coco sites in the corresponding profiles (waterfront H0, H1 middle and back H2). Boca Zacate, Térraba Sierpe National Wetland, Costa Rica. 2008-2013.

una cantidad importante de sedimentos finos como limos y arcillas la mayor parte del año. Sin embargo, en la época lluviosa prevalecen las arenas durante las grandes crecidas del río Grande de Térraba.

Los esteros Brujo y Bocón poseen una influencia directa con la línea de costa, favoreciendo el depósito de sedimentos directamente al mar. La posición de la boca de ambos esteros hacia el interior de Boca Zacate acelera el depósito de sedimentos provenientes del río Grande de Térraba por parte de las corrientes en este sector. El estero Camíbar presentó la menor tasa de sedimentación pues no esta sujeto al efecto directo del río Grande de Térraba y al impacto del oleaje, minimizando su contribución en los procesos de cambio costero en Boca Zacate. 
La redistribución litoral de los sedimentos por parte de las corrientes marinas, las mareas y el oleaje (Lizano, 2012), favorece una composición mayor de un $50 \%$ de arenas, cuyo depósito propicia la formación de las islas barrera hacia el frente del delta del río Grande de Térraba (Espinoza et al, 2005). En los sectores del Brujo, Bocón e isla Coco es notoria la progradación con una alta predominancia de arena, especialmente en las partes superiores de la línea de costa. La arena acumulada sobrepasa la altura de las raíces de mangle, obstruyendo las lenticelas e impidiendo el intercambio de oxígeno entre el aire y las estructuras internas del árbol durante la marea baja, provocando la muerte del fuste (Blasco et al., 1996 \& Ellison, 1998).

El bosque de mangle en los sectores superiores de la costa en Boca Zacate, se encuentra en proceso de deterioro, mientras que los frentes de playa, también altamente arenosos, poseen relictos de vegetación de manglar que evidencian procesos de anteriores erosiones. Con la muerte y la descomposición de la vegetación se favorece la erosión del terreno debido a la baja cohesión de la arena ante el impacto de las olas, lo que ha conllevado a una pérdida del bosque de mangle, disminuyendo el área emergida de Boca Zacate y la capacidad de capturar, retener y almacenar sedimentos por parte de los árboles, provocando un retroceso de la línea de costa como se evidencia en el presente estudio.

Los manglares se caracterizan por ser eficientes sistemas en atrapar y reciclar nutrimentos (Furukawa et al., 1996; Middelburg, Nieuwenhuize, Slim, \& Ohowa, 1996), sin embargo, su dinámica está regulada por el tipo de sustrato, ámbito de mareas, elevación, tipo de bosque y precipitación (Alongi, Boto, \& Robertson, 1992; Middelbug et al., 1996). En bosques de mangle deteriorados, estos procesos de reciclaje, retención e incorporación por parte de las raíces no se cumplen en su totalidad (Ellison, 1998). En un manglar como Boca Zacate donde predominan las arenas, la retención de nutrimentos por parte de este sustrato es deficiente, pues el tamaño del grano no permite una retención completa de los nutrimentos en el sustrato, favoreciendo el lavado de estas sustancias fuera del manglar y por consiguiente, el no aprovechamiento por parte de las raíces, lo que ocasiona un deterioro general del bosque de mangle.

Los resultados reflejan que en los sitios donde existe una predominancia de arenas, la concentración de nutrimentos disminuye; por esta razón, la zona supralitoral presenta la menor concentración de todos los elementos analizados. En sustratos más profundos, la concentración de los elementos químicos aumenta, lo cual indica la presencia de fracciones de arcilla y limo que permiten acumular estos compuestos.

Estos procesos costeros en la zona del HNTS, confirman que específicamente en Boca Zacate, la descarga de sedimentos por parte del río Grande de Térraba y su posterior transporte y distribución por las corrientes, el oleaje y las mareas (Espinoza et al., 2005; Ortiz, 2008; Lizano, 2012), no reúnen las condiciones para la sobrevivencia y establecimiento de manglares en la zona. Sin embargo, en áreas internas protegidas de la energía litoral y fuera de la influencia directa del río Grande de Térraba prevalece la progradación de sedimentos más finos y favorece la regeneración del bosque.

En conclusión, el aporte significativo de sedimento, especialmente arena por parte del río Grande de Térraba, ha favorecido la progradación de playas arenosas, situación que paralela a la erosión de la línea de costa han reducido la cobertura de los bosques de mangle, evidente por la presencia de árboles muertos en Boca Zacate. Este panorama propone la necesidad de plantear lineamientos para la conservación de este espacio, por ejemplo la recuperación y protección de una zona de amortiguamiento en el límite continental del HNTS, de manera que se brinde un espacio para la recuperación del ecosistema, en conjunto con buenas prácticas de uso y conservación del suelo en los terrenos a nivel de cuenca. 


\section{AGRADECIMIENTOS}

Los autores agradecen a la Vicerrectoría de Investigación de la Universidad de Costa Rica por el apoyo a esta investigación a través del Programa Institucional de la Península de Osa, bajo el marco del Proyecto No. 808 A7 084 adscrito al CIMAR y la Escuela de Biología. Al Proyecto Hidroeléctrico El Diquís, del Instituto Costarricense de Electricidad durante los muestreos en el sitio de estudio. Los asistentes Andrey Villalobos Jiménez, Tito Isaac Sancho y Desta Montcalm por el trabajo en el campo y el procesamiento de las muestras en el laboratorio, al geógrafo Carlos Cordero Vega por su aporte en la elaboración del material cartográfico y a cuatro revisores anónimos que aportaron sugerencias valiosas para el artículo.

\section{RESUMEN}

En los últimos sesenta años han ocurrido cambios en el Humedal Nacional Térraba-Sierpe, Costa Rica. Los cambios en la geomorfología costera están asociados a la erosión o acreción de sedimentos, lo que ha favorecido la eliminación de sectores de bosque o a la formación de bancos de sedimentos que han sido colonizados por árboles de mangle. El estudio se realizó en los sectores Bocón, Brujo e isla Coco en Boca Zacate, Humedal Nacional TérrabaSierpe, Costa Rica, entre 2008-2013, cuyo objetivo consiste en analizar el aporte de sedimentos y las implicaciones en la cobertura del bosque de mangle en el sector de Boca Zacate. Es este estudio se realizó un análisis multitemporal de la morfología litoral con fotografías aéreas e imágenes satelitales de 1948, 1960, 1974, 1978, 1984, 1992 y 2011. Y además, se cuantificó la tasa de sedimentación mensual ( $\mathrm{g} / \mathrm{cm}^{2} /$ día) en los canales de los estuarios, la composición granulométrica y la concentración de elementos químicos en los sedimentos $\left(\mathrm{mgL}^{-1}\right)$ en época seca y lluviosa durante los años de estudio. Los resultados indican que Boca Zacate ha mantenido en los últimos 60 años una tendencia hacia la erosión, perdiendo un $10.6 \%$ de su espacio emergido entre 1948 y 2011 y aproximadamente un $8.9 \%$ de su cobertura forestal; también ha experimentado una acreción en la zona de la isla Coco, uniéndose al continente por este sector. La tasa de sedimentación cuantificada en los esteros presentó diferencias con respecto al sitio y a la época. La mayor cantidad se presentó en el estero el Brujo y la menor cantidad en el estero Camíbar. Con respecto a la granulometría, el tipo de sedimento dominante es la arena, seguido por la arcilla y en menor cantidad el limo en todos los sitios de estudio. La mayor distribución de los elementos químicos corresponde al magnesio, calcio, potasio; mientras que el manganeso, hierro, aluminio, fósforo, zinc y cobre se encontraron en menor cantidad. El transporte, composición y cantidad de sedimentos en Boca Zacate aportados por el río Grande de Térraba, asociados a las corrientes, mareas y oleaje, son factores determinantes en los cambios que han ocurrido en el litoral de la Boca, conllevando a un paisaje de bosque de mangle muerto en la zona. Por consiguiente, este tipo de análisis es importante para la recomendación de lineamientos y acciones en la conservación y la gestión integrada del espacio marino-costero en el Humedal Nacional Térraba Sierpe.

Palabras clave: morfodinámica litoral, sedimentación litoral, bosque de manglar, Térraba-Sierpe, Costa Rica.

\section{REFERENCIAS}

Adams, A. E., MacKenzie, W. S., \& Guilford, C. (1984). Atlas of Sedimentary Rocks under the Microscope. Inglaterra: Longman Group Ltd. Essex.

Alongi, D. (2002). Present state and future of the world's mangrove forests. Environmental Conservation, 3, 331-349.

Alongi, D. M., Boto, K. G., \& Robertson, A. (1992). Nitrogen and phosphorus cycles. In A. I. Robertson, \& D. M. Alongi (Eds.), Tropical mangrove ecosystems (pp. 251-292). Washington, DC: American Geophysical Union.

Arche, A. (1989). Deltas. In A. Arche (Ed.), Sedimentología (pp. 395-449). Madrid, España: Consejo Superior de Investigación Científicas.

Bird, E., \& Barson, M. (1977). Measurement of physiographic changes on mangrove-fringed estuaries coastlines. Marine Research Indonesia, 18, 73-80.

Blanco, J. F., Estrada, L. F., \& Urrego, L. E. (2012). Ecosystem-Wide Impacts of Deforestation in Mangroves: The Uraba Gulf (Colombian Caribbean) Case Study. ISRN Ecology, 1-14.

Blasco, F., Saenger, P., \& Janodet, E. (1996). Mangroves as indicators of coastal change. Catena, 27, 167-178.

Cárdenes, G. (2003). Evolución de los sistemas sedimentarios costero y aluvial de la región de Parrita, Pacífico Central de Costa Rica. Revista Geológica de América Central, 31, 45-59.

Cedeño, B., López, A., Villalobos, E., \& Hernández, A. (2012). Ejes modificadores de las condiciones biofísicas de la Cuenca del Río Grande de Térraba. Revista Geográfica de América Central, 48, 95-116.

Denyer, P., Cárdenes, G., \& Kruse, S. (2004). Registro histórico y evolución de la barra arenosa de Puntarenas, Golfo de Nicoya, Costa Rica. Revista Geológica de América Central, 31, 45-59. 
Elliot, T. (1991). Deltas. In H. Reading (Ed.). Sedimentary Environments and Facies (2da ed.). Londres, Inglaterra: Oxford.

Ellison, J. C. (1998). Impacts of sediment burial on mangroves. Marine Pollution Bulletin, 37, 420-426.

Espinoza, J., Rojas, E., Aguilar, J., Gutiérrez, V., Granados, G., \& Rodríguez, H. (2005). Análisis Sedimentológico del Delta Térraba. Campaña Geológica 2005. San José: Universidad de Costa Rica.

Furukawa, K., \& Wolanski, E. (1996). Sedimentation in mangrove forest. Mangroves and salt marshes, 1, $3-10$.

González, C., Urrego, L., Martínez, J., Polanía, J., \& Yokoyama, Y. (2010). Mangrove dynamics in the southwestern Caribbean since the "Little Ice Age": A history of human and natural disturbances. The Holocene, 20, 849-861.

Gutiérrez, E. M. (2008). Geomorfología. Madrid, España: Pearson/Prentice Hall.

Jiménez, J. A., Lugo, A. E., \& Cintrón, G. (1985). Tree mortality in mangrove forests. Biotropica, 17, 177-185.

Kathiresan, K. (2003). How do mangrove forest induce sedimentation? Revista Biología Tropical, 51, 355-360.

Lizano, O., \& Salas, M. (2001). Variaciones geomorfológicas en los últimos 50 años de la Isla Damas, Quepos, Costa Rica. Revista de Biología Tropical, 49, 171-177.

Lizano, M., \& Lizano, O. (2010). Creación de escenarios de inundación en la Ciudad de Puntarenas ante el aumento del nivel del mar. InterSedes, XI(21), 215-229.

Lizano, R. O. G. (2012). Estudio oceanográfico costero en las desembocaduras del Humedal Nacional Térraba
Sierpe (HNTS). Informe Técnico preparado para El Instituto Costarricense de Electricidad (ICE), Proyecto Hidroeléctrico El Diquís (PHED).

Lizano, O. G., Alfaro, E. J., \& Salazar, A. (2012). Un método para evaluar el enriquecimiento de metales en sedimentos marinos en Costa Rica. Revista de Biología Tropical, 60(Suppl. 2), 197-211.

Lizano, O. (2013). Erosión en las costas de Costa Rica. Módulo de Información Oceanográfica. Recuperado de http://www.miocimar.ucr.ac.cr/erosioncostarica.

Middelburg, J., Nieuwenhuize, J., Slim, F., \& Ohowa, B. (1996). Sediment biogeochemistry in an East African mangrove forest (Gazi Bay, Kenya). Biogeochemistry, 34, 133-155.

Mora, S. C. (1989). Extent and social-economic significance of slope instability in Costa Rica. In Landslides: Extent and Economic Significance. Proceedings of the 28th International Geological Congress: Symposium on Landslides (pp. 93-99). Balkema: Rotterdam.

Olsen, R., Cole, C. V., Watanabe, F. S., \& Dean, L. A. (1954). Estimation of available phosphorus in soils by extraction with sodium bicarbonate. USDA Circ. 939 USDA, Washington, DC.: Government printing office.

Ortiz, M. E. (2008). Cambios geomorfológicos en el litoral Caribe y Pacífico de Costa Rica. Caso del Complejo Déltico de Sierpe. Revista Forestal Mesoamericana Kurú, 5, 15 .

Rojas, N. (2011). Cuenca Río Grande de Térraba. San José, Costa Rica.

Woodroffe, C. (1992). Mangrove Sediments and Geomorphology. In D. Alongi, \& E. Robertson (Eds.), Coastal and Estuarine Studies, Tropical Mangrove Ecosystems (pp. 51-70). Washington, DC: American Geophysical Union. 
